\title{
Animal Mix Antigen IgG Antibody Measurement
}

National Cancer Institute

\section{Source}

National Cancer Institute. Animal Mix Antigen Ig G Antibody Measurement. NCI

Thesaurus. Code C130113.

A measurement of the animal mix antigen Ig $G$ antibody in a biological specimen. 